TRANSACTIONS OF THE

AMERICAN MATHEMATICAL SOCIETY

Volume 351, Number 2, February 1999, Pages 837-856

S 0002-9947(99)02035-8

\title{
DISTRIBUTION SEMIGROUPS AND ABSTRACT CAUCHY PROBLEMS
}

\author{
PEER CHRISTIAN KUNSTMANN
}

\begin{abstract}
We present a new definition of distribution semigroups, covering in particular non-densely defined generators. We show that for a closed operator $A$ in a Banach space $E$ the following assertions are equivalent: (a) $A$ generates a distribution semigroup; (b) the convolution operator $\delta^{\prime} \otimes I-\delta \otimes A$ has a fundamental solution in $\mathcal{D}^{\prime}(L(E, D)$ ) where $D$ denotes the domain of $A$ supplied with the graph norm and $I$ denotes the inclusion $D \rightarrow E$; (c) $A$ generates a local integrated semigroup. We also show that every generator of a distribution semigroup generates a regularized semigroup.
\end{abstract}

\section{INTRODUCTION}

Distribution semigroups in Banach spaces and their generators were introduced by J. L. Lions in [16] as a generalization of $C_{0}$-semigroups and their generators. Like the generators of $C_{0}$-semigroups the generators of the distribution semigroups in [16] are densely defined. It was then shown in [16] that the densely defined closed linear operators $A$ that generate a distribution semigroup are exactly those for which the convolution operator $\delta^{\prime} \otimes I-\delta \otimes A$ where $I$ denotes the inclusion $D(A) \rightarrow E$ has a fundamental solution.

Treating Cauchy problems as convolution equations has turned out to be very useful (see [6], [12], [13] and [14]). But in doing so the assumption that $D(A)$ is dense in $E$ is unnecessary in most of the results.

On the other hand in the last years there has been an interest in dealing with operators that are not densely defined, starting perhaps with [7]. Reasons for this interest may be that it might not be so easy to decide whether a given operator is densely defined and the difficulties that arise when passing from $E$ to $E^{*}$ or $l^{\infty}(E)$.

So the question how the theory of distribution semigroups introduced in [16] can be extended to cover non-densely defined generators in such a way that the above characterization via fundamental solutions holds seems natural.

It turns out that this indeed is possible in a way that even simplifies the original definitions given in [16]. Moreover, the local strongly continuous representations (see section 4) of the distribution semigroups thus obtained are exactly the local integrated semigroups (for the distribution semigroups in the sense of [16] a relation to local integrated semigroups has been obtained in [23], Theorem 5.6; see also [15], Theorem 7.6 or [2], Theorem 7.2). So the theory presented here

Received by the editors October 17, 1995 and, in revised form, February 6, 1997

1991 Mathematics Subject Classification. Primary 47D03, 34G10, 47A10, 46F10.

(C)1999 American Mathematical Society 
can be viewed as a unified approach to treating Cauchy problems via fundamental solutions, distribution semigroups or local integrated semigroups - without any denseness assumptions.

The first section deals with preliminaries; in the second we introduce distribution semigroups (DSGs) and more generally pre-DSGs. Section 3 is devoted to the study of the generator of a DSG and the relationship to fundamental solutions, and section 4 deals with local representations of a DSG as an iterated derivative of a strongly continuous function and covers the relationship to local integrated semigroups. In section 5 we show that every generator of a DSG generates a regularized semigroup, a result that even in the dense case seems not to have been stated elsewhere, while it is clear that the reverse implication is false in general (see $[8])$.

The author wants to thank V. Wrobel for many helpful discussions, L. Weis for preprints of [2] and [10] and V. Keyantuo for a copy of [15].

\section{Preliminaries}

We will use the following notations. $\mathbb{R}$ and $\mathbb{C}$ denote the real and complex scalar field. $\mathbb{N}$ denotes the set of all positive integers and $\mathbb{N}_{0}$ the set of all non-negative integers.

$\mathcal{D}$ denotes the space of all $C^{\infty}$-functions $\mathbb{R} \rightarrow \mathbb{C}$ with compact support supplied with the usual inductive limit topology. We denote by $\mathcal{D}_{B}$ the subspace of functions in $\mathcal{D}$ with support in $B \subset \mathbb{R}$ and let $\mathcal{D}_{0}:=\mathcal{D}_{[0, \infty[}$ for short. Further, let $*_{0}$ denote the convolution-like mapping given by

$$
f *_{0} g(t):=\int_{0}^{t} f(s) g(t-s) d s .
$$

Notice that $*_{0}: \mathcal{D} \times \mathcal{D} \rightarrow \mathcal{D}$ is a bilinear, separately continuous mapping which is moreover associative, symmetric and coincides on $\mathcal{D}_{0} \times \mathcal{D}_{0}$ with the usual convolution $*$. If $f$ is differentiable, then $f *_{0} g$ is, too, and

$$
\left(f *_{0} g\right)^{\prime}=f^{\prime} *_{0} g+f(0) g .
$$

By a regularizing sequence we mean a sequence $\left(\rho_{n}\right)$ in $\mathcal{D}_{0}$ obtained by $\rho_{n}:=n \rho(n \cdot)$ from a $\rho$ with $\int \rho d t=1$.

If $A$ is a linear operator in a Banach space $E$ we denote the resolvent set of $A$, i.e. the set of all $\lambda \in \mathbb{C}$ such that $\lambda-A: D(A) \rightarrow E$ is bijective, by $\rho(A)$, and for $\lambda \in \rho(A)$ the resolvent operator $(\lambda-A)^{-1}$ by $R(\lambda, A)$. We denote the kernel of a linear mapping $T$ by $\operatorname{Kern} T$ and the image of $T$ by $\operatorname{Im} T$.

For every Banach space $X$ we denote by $\mathcal{D}^{\prime}(X)$ the space of all continuous linear mappings $\mathcal{D} \rightarrow X$ supplied with the topology of uniform convergence on the bounded subsets of $\mathcal{D}$. We denote the set of all $T \in \mathcal{D}^{\prime}(X)$ with $\operatorname{supp} T \subset[0, \infty[$ supplied with the induced topology by $\mathcal{D}_{0}^{\prime}(X)$. Notice that $\mathcal{D}_{0}^{\prime}(X)=\mathcal{D}_{0}^{\prime} \widehat{\otimes}_{\pi} X$ since $\mathcal{D}_{0}^{\prime}$ is nuclear, the set of all elementary tensors is dense and $\mathcal{D}_{0}^{\prime}(X)$ is complete.

The definition and properties of the convolution of vector-valued distributions can be found in [20] but the situation there is fairly general and the proofs are quite involved whereas the elementary approach in [14], chapter 8, does not give the full continuity property. For the convenience of the reader we will therefore state a special version of Proposition 34 in [20], part II, sufficient for our purposes, and give a proof which in this special situation is quite simple though the argument is essentially the same as in [20]. 
Proposition 1.1. Suppose $X, Y$ and $Z$ are Banach spaces and $b: X \times Y \rightarrow Z$ is bilinear and continuous. Then there is a unique bilinear, separately continuous mapping

$$
*_{b}: \mathcal{D}_{0}^{\prime}(X) \times \mathcal{D}_{0}^{\prime}(Y) \rightarrow \mathcal{D}_{0}^{\prime}(Z)
$$

such that

$$
(S \otimes x) *_{b}(T \otimes y)=S * T \otimes b(x, y)
$$

holds for all $S, T \in \mathcal{D}_{0}^{\prime}$ and $x \in X, y \in Y$. This mapping is moreover continuous.

Proof. It is clearly sufficient to prove the case $Z=X \widehat{\otimes}_{\pi} Y$ and $b:(x, y) \mapsto x \otimes y$. Since uniqueness is clear from the denseness of elementary tensors all we have to show is that the bilinear mapping

$$
\left(\mathcal{D}_{0}^{\prime} \otimes_{\pi} X\right) \times\left(\mathcal{D}_{0}^{\prime} \otimes_{\pi} Y\right) \rightarrow \mathcal{D}_{0}^{\prime} \otimes_{\pi}\left(X \otimes_{\pi} Y\right)
$$

which is given by (2) is continuous. This is equivalent to its linearization

$$
\left(\mathcal{D}_{0}^{\prime} \otimes_{\pi} X\right) \otimes_{\pi}\left(\mathcal{D}_{0}^{\prime} \otimes_{\pi} Y\right) \rightarrow \mathcal{D}_{0}^{\prime} \otimes_{\pi}\left(X \otimes_{\pi} Y\right)
$$

being continuous. Since the convolution of scalar-valued distributions $*: \mathcal{D}_{0}^{\prime} \times \mathcal{D}_{0}^{\prime} \rightarrow$ $\mathcal{D}_{0}^{\prime}$ is continuous (see [19]), its linearization $\hat{*}: \mathcal{D}_{0}^{\prime} \otimes_{\pi} \mathcal{D}_{0}^{\prime} \rightarrow \mathcal{D}_{0}^{\prime}$ is continuous, and we are done by the associativeness of $\otimes_{\pi}$.

The convolution of Proposition 1.1 is also associative under obvious assumptions on the bilinear mappings involved since $\otimes_{\pi}$ is associative.

\section{Definition and Elementary PRoperties of PRE-DSGs}

Throughout this section $E$ will denote a Banach space.

Definition 2.1. A pre- $D S G$ is an element $G \in \mathcal{D}_{0}^{\prime}(L(E))$ with

$$
\forall \varphi, \psi \in \mathcal{D}: G(\varphi) G(\psi)=G\left(\varphi *_{0} \psi\right) .
$$

We call a pre-DSG non-degenerate or a $D S G$ for short if

$$
\mathcal{N}(G):=\bigcap\left\{\operatorname{Kern} G(\varphi): \varphi \in \mathcal{D}_{0}\right\}=\{0\} .
$$

We call (by abuse of language) $\mathcal{N}(G)$ the kernel space of $G$. A pre-DSG $G$ is called dense if

$$
\mathcal{R}(G):=\bigcup\left\{\operatorname{Im} G(\varphi): \varphi \in \mathcal{D}_{0}\right\} \text { is dense in } E .
$$

In [16] the definition of a distribution semigroup is different from the one given above, and we will consider the differences in Remark 3.13. The following lemma makes clear the structure of a pre-DSG on its kernel space.

Lemma 2.2. If $G$ is a pre-DSG in $E$, then $\mathcal{N}(G)$ is a closed subspace of $E$. Let $F:=\mathcal{N}(G)$ and $H:=\left.G(\cdot)\right|_{F}$. Then there is a $T \in L(F)$ and an integer $m \geq 0$ such that

$$
H=\sum_{j=0}^{m} \delta^{(j)} \otimes(-1)^{j} T^{j+1}
$$

and $T^{m+2}=0$. We call $T$ the kernel operator of $G$. 
Proof. $F$ is invariant under $G$ because of (3), so $H$ is well defined. We have $H \in \mathcal{D}^{\prime}(L(F))$ with supp $H \subset\{0\}$. So there is an integer $m \geq 0$ and operators $T_{0}, \ldots, T_{m} \in L(F)$ with

$$
H=\sum_{j=0}^{m} \delta^{(j)} \otimes T_{j}
$$

Because of (3) all $T_{j}$ commute, and for all $\varphi, \psi \in \mathcal{D}$ we get

$$
\sum_{j=0}^{m} \sum_{k=0}^{m} \delta^{(j)}(\varphi) \delta^{(k)}(\psi) T_{j} T_{k}=-\sum_{j=1}^{m} \sum_{k=0}^{j-1} \delta^{(k)}(\varphi) \delta^{(j-1-k)}(\psi) T_{j},
$$

where we have used the equality

$$
\left(\varphi *_{0} \psi\right)^{(j)}(0)=\sum_{k=0}^{j-1} \varphi^{(k)}(0) \psi^{(j-1-k)}(0) .
$$

If we choose $\varphi, \psi \in \mathcal{D}$ with

$$
\delta^{(j)}(\varphi) \delta^{(\nu)}(\psi)=\delta_{0 j} \delta_{k \nu},
$$

we get for each $k \in\{0, \ldots, m\}$ :

$$
T_{0} T_{k}=-\sum_{j=1}^{m} \delta^{(j-1)}(\psi) T_{j}= \begin{cases}-T_{k+1}, & k<m, \\ 0, & k=m,\end{cases}
$$

from which the claim follows by induction.

On the other hand we can use Lemma 2.2 to construct pre-DSGs.

Example 2.3. Let $T \in L(E)$ be nilpotent with order $n$. Then

$$
G(\psi) x:=\sum_{j=0}^{n-2} \psi^{(j)}(0) T^{j+1} x
$$

for all $\psi \in \mathcal{D}$ and $x \in E$ defines a pre-DSG $G$ in $E$ with $\mathcal{N}(G)=E$. Indeed, since $T^{n}=0$ we have for $\varphi, \psi \in \mathcal{D}$

$$
G(\varphi) G(\psi)=\sum_{j=0}^{n-3} \sum_{k=0}^{n-3-j} \varphi^{(j)}(0) \psi^{(k)}(0) T^{j+k+2}
$$

and

$$
\begin{aligned}
G\left(\varphi *_{0} \psi\right) & =\sum_{j=1}^{n-2} \sum_{k=0}^{j-1} \varphi^{(k)}(0) \psi^{(j-1-k)}(0) T^{j+1}=\sum_{j=0}^{n-3} \sum_{k=0}^{j} \varphi^{(k)}(0) \psi^{(j-k)}(0) T^{j+2} \\
& =\sum_{k=0}^{n-3} \sum_{\nu=0}^{n-3-k} \varphi^{(k)}(0) \psi^{(\nu)}(0) T^{k+\nu+2} .
\end{aligned}
$$

Lemma 2.4. Suppose $G$ is a pre-DSG in $E$. Let $F:=E / \mathcal{N}(G)$ and $q$ be the quotient map. Then $H(\psi) q(x):=q(G(\psi) x)$ defines an element $H(\psi) \in L(F)$ with $q \circ G(\psi)=H(\psi) \circ q$ for all $\psi \in \mathcal{D}$. The mapping $H: \psi \mapsto H(\psi)$ is a DSG in $F$. 
Proof. Let $\psi \in \mathcal{D}$. We show that $H(\psi)$ is well defined. So let $x \in \mathcal{N}(G)$ and $\varphi \in \mathcal{D}_{0}$. Using (3) we get

$$
G(\varphi) G(\psi) x=G(\psi) G(\varphi) x=G(\psi) 0=0,
$$

i.e. $G(\psi) x \in \mathcal{N}(G)$. So $H(\psi)$ is well defined and we have $\|H(\psi)\| \leq\|G(\psi)\|$. So $H \in \mathcal{D}_{0}^{\prime}(L(F))$ and obviously $H$ is a pre-DSG.

Let now $y \in \mathcal{N}(H)$ and $x \in y$. Then $G(\varphi) x \in \mathcal{N}(G)$ for all $\varphi \in \mathcal{D}_{0}$. Supposing

$$
\mathcal{N}(G) \cap\langle\mathcal{R}(G)\rangle=\{0\}
$$

we get $G(\varphi) x=0$ for all $\varphi \in \mathcal{D}_{0}$, i.e., $x \in \mathcal{N}(G)$, which implies $y=\mathcal{N}(G)=0_{F}$. So we have to prove (6). Let $z=\sum_{j} G\left(\varphi_{j}\right) x_{j} \in \mathcal{N}(G) \cap\langle\mathcal{R}(G)\rangle$. We choose a regularizing sequence $\left(\rho_{n}\right)$ in $\mathcal{D}_{0}$. Because of $(3)$ and $z \in \mathcal{N}(G)$ we get

$$
z=\lim _{n \rightarrow \infty} \sum_{j} G\left(\rho_{n} * \varphi_{j}\right) x_{j}=\lim _{n \rightarrow \infty} G\left(\rho_{n}\right) z=0,
$$

and (6) is proved.

Remark 2.5. Let $G$ be a pre-DSG. Then $\overline{\mathcal{R}(G)}=\overline{\langle\mathcal{R}(G)\rangle}$.

Proof. We only have to show that the right side is a subset of the left side. So let $y=\sum_{j} G\left(\varphi_{j}\right) x_{j} \in\langle\mathcal{R}(G)\rangle$. Choose a regularizing sequence $\left(\rho_{n}\right)$ in $\mathcal{D}_{0}$. Using (3) we get

$$
y=\lim _{n \rightarrow \infty} \sum_{j} G\left(\rho_{n} * \varphi_{j}\right) x_{j}=\lim _{n \rightarrow \infty} G\left(\rho_{n}\right) y \in \overline{\mathcal{R}(G)} .
$$

The next lemma shows how to obtain a dense pre-DSG from a given pre-DSG.

Lemma 2.6. Let $G$ be a non-dense pre-DSG in E. Denote by $F$ the closure of $\mathcal{R}(G)$ in $E$. Then $\left.G(\psi)\right|_{F} \in L(F)$ for each $\psi \in \mathcal{D}$ and $H:=\left.G(\cdot)\right|_{F}$ is a dense pre-DSG in $F$.

Proof. Let $\psi \in \mathcal{D}$. Because of $(3)\langle\mathcal{R}(G)\rangle$ is invariant under $G(\psi)$, so $F$ is also invariant. This implies $H \in \mathcal{D}_{0}^{\prime}(L(F))$. Obviously $H$ is a pre-DSG in $F$. To prove denseness we show $\mathcal{R}(G) \subset \overline{\mathcal{R}(H)}$. Let $\left(\rho_{n}\right)$ be a regularizing sequence in $\mathcal{D}_{0}$ and $y=G(\varphi) x \in \mathcal{R}(G)$. Using (3) we get

$$
y=\lim _{n \rightarrow \infty} G\left(\rho_{n} * \varphi\right) x=\lim _{n \rightarrow \infty} G\left(\rho_{n}\right) y=\lim _{n \rightarrow \infty} H\left(\rho_{n}\right) y \in \overline{\mathcal{R}(H)} .
$$

The following lemma treats the dualization of pre-DSGs. Here $M^{\circ} \subset E^{*}[E]$ denotes the polar of a set $M \subset E\left[E^{*}\right]$.

Lemma 2.7. Let $G$ be a pre-DSG in $E$. Then $G(\cdot)^{*}$ is a pre-DSG in $E^{*}$ and we have

If $E$ is reflexive, then

$$
\mathcal{N}\left(G(\cdot)^{*}\right)=\overline{\mathcal{R}(G)}^{\circ} .
$$

$$
\mathcal{N}(G)=\overline{\mathcal{R}\left(G(\cdot)^{*}\right)} .
$$

So $G(\cdot)^{*}$ is a DSG in $E^{*}$ if and only if $G$ is a dense pre-DSG in $E$. If $E$ is reflexive, then $G$ is a $D S G$ in $E$ if and only if $G(\cdot)^{*}$ is a dense pre-DSG in $E^{*}$.

Proof. It is clear that $G(\cdot)^{*} \in \mathcal{D}_{0}^{\prime}\left(L\left(E^{*}\right)\right)$ and (3) holds. The first equation follows from

$$
\left\langle x^{*}, G(\varphi) x\right\rangle=\left\langle G(\varphi)^{*} x^{*}, x\right\rangle
$$

for all $x \in E, x^{*} \in E^{*}$ and $\varphi \in \mathcal{D}_{0}$ using Remark 2.5. To prove the second equation remember the fact $G(\cdot)^{* *}=G$ for $E$ reflexive and use the first equation. 
We give an example, both of a non-dense DSG and a dense pre-DSG that is not a DSG, in a separable Hilbert space. The example is a modification of an example in [17].

Example 2.8. Let $m \in \mathbb{N}$. Denote by $H^{m}$ the completion of $C^{m}[0,1]$ with respect to the norm

$$
f \mapsto\|f\|_{H^{m}}:=\sum_{\alpha \leq m}\left\|f^{(\alpha)}\right\|_{L^{2}} .
$$

Then $H^{m}$ is a separable Hilbert space. We define for $\varphi \in \mathcal{D}$ and $f \in H^{m}$

$$
G(\varphi) f:=\left(\varphi *_{0} f\right) 1_{[0,1]} .
$$

Then $G$ is a pre-DSG in $H^{m}$. Using a regularizing sequence we get $\mathcal{N}(G)=\{0\}$. On $C^{m}[0,1]$ the norm $\|\cdot\|_{H^{m}}$ is equivalent to the norm

$$
\|f\|:=\sum_{\alpha<m}\left\|f^{(\alpha)}\right\|_{\infty}+\left\|f^{(m)}\right\|_{L^{2}}
$$

Thus $\mathcal{R}(G) \subset\left\{f \in H^{m}: \forall \alpha<m: f^{(\alpha)}(0)=0\right\}$. Since $m \geq 1, G$ is non-dense. By Lemma $2.7 G(\cdot)^{*}$ is a dense pre-DSG in the separable Hilbert space $\left(H^{m}\right)^{*}$, but $G(\cdot)^{*}$ is not a DSG.

\section{The Generator of A DSG}

Denote by $\mathcal{E}_{0}^{\prime}$ the set of all elements of $\mathcal{E}^{\prime}$ with support contained in $[0, \infty[$. We follow the idea in [16] while modifying the definition for our purposes. Let for this section $G$ be a DSG in $E$.

Definition 3.1. Let $T \in \mathcal{E}_{0}^{\prime}$. Then

$$
\tilde{G}(T):=\left\{(x, y) \in E \times E: \forall \varphi \in \mathcal{D}_{0}: G(T * \varphi) x=G(\varphi) y\right\} .
$$

(Note that $T * \varphi \in \mathcal{D}_{0}$ for each $\varphi \in \mathcal{D}_{0}$.)

For $T=-\delta^{\prime}$ this definition has been used in [21] in a locally convex setting but for $G$ dense.

Remark 3.2. a) Using a regularizing sequence and (3) we see that in the definition of $\tilde{G}(T) \mathcal{D}_{0}$ can be replaced by $\mathcal{D}_{[0, \epsilon]}$ for each $\epsilon>0$.

b) Because of $(4) \tilde{G}(T)$ is a function. It is easy to see that $\tilde{G}(T)$ is a closed linear operator in $E$ for each $T \in \mathcal{E}_{0}^{\prime}$.

c) Obviously $\tilde{G}(\delta)=I d_{E}$ and $\tilde{G}\left(\left.\psi\right|_{[0, \infty[}\right)=G(\psi)$ for all $\psi \in \mathcal{D}$.

Because of Remark $3.2 \mathrm{c}$ ) we denote the operator $\tilde{G}(T)$ by $G(T)$. We define the generator of $G$ to be the linear operator $G\left(-\delta^{\prime}\right)$.

Definition 3.3. The generator $A$ of $G$ is the closed linear operator $A:=G\left(-\delta^{\prime}\right)$.

We will give some examples. The first is a modification of an example in [10].

Example 3.4. Let $\Omega:=\left\{x+i y: x>0, y>e^{x}\right\}$ and $E:=\left(H^{\infty}(\Omega) \cap C_{b}(\bar{\Omega}),\|\cdot\|_{\infty}\right)$. Let $A$ be defined by $(A f)(z):=z f(z)$ with maximal domain. Then $A$ generates a DSG $G$ in $E$ which is given by

$$
(G(\varphi) f)(z):=\int_{0}^{\infty} \varphi(t) e^{t z} d t f(z)
$$

for all $\varphi \in \mathcal{D}$ and $f \in E$. Note that there are no non-trivial exponentially bounded solutions of $u^{\prime}=A u$ in this example. 
In the next example $A$ is a differential operator. A finite version with $\bigcap_{k=0}^{m}$ instead of $\bigcap_{k \geq 0}$ appeared in [10], Example 5.20.

Example 3.5. Let

$$
E:=\left\{f \in \bigcap _ { k \geq 0 } C ^ { k } \left[k, \infty\left[: f(0)=0,\|f\|_{E}:=\sup _{k} \sup _{t \geq k}\left|f^{(k)}(t)\right|<\infty\right\} .\right.\right.
$$

Then letting $G(\varphi) f:=\left.\varphi\right|_{[0, \infty[} * f$ for $\varphi \in \mathcal{D}$ and $f \in E$ defines a DSG $G$ in $E$ whose generator is $-d / d x$ with maximal domain.

Indeed, if $\varphi \in \mathcal{D}$ with $\operatorname{supp} \varphi \subset]-\infty, n]$, then

$$
\left(\left.\varphi\right|_{[0, \infty[} * f\right)^{(k)}(t)=\left(\left.\varphi^{(k)}\right|_{[0, \infty[}\right) * f(t)+\sum_{j=0}^{k-1} \varphi^{(j)}(0) f^{(k-1-j)}(t),
$$

for all $f \in E, k \in \mathbb{N}_{0}$ and $t \geq k$. So we only have to estimate $\left\|\left.\varphi\right|_{[0, \infty[} * f\right\|_{E}$. Since $\left.\operatorname{supp} \varphi\right|_{[0, \infty[} \subset[0, n]$ and $\left.f\right|_{[k-n, \infty[}$ is a $C^{k-n}$-function we get for $k>n$ and $t \geq k$

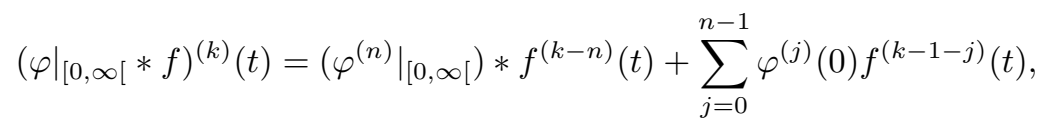

which implies

$$
\left\|\left.\varphi\right|_{[0, \infty[} * f\right\|_{E} \leq\left(\sup _{k \leq n}\left\|\varphi^{(k)}\right\|_{L^{1}}+\sum_{j=0}^{n-1}\left|\varphi^{(j)}(0)\right|\right)\|f\|_{E} .
$$

So $G \in \mathcal{D}_{0}^{\prime}(L(E))$, and clearly $G$ is a pre-DSG. By Laplace transformation we see that $G(\varphi)$ is injective for each $\varphi \in \mathcal{D}_{0} \backslash\{0\}$, so $\mathcal{N}(G)=\{0\}$ and $G$ is a DSG. The verification of the fact that $A$ is the generator is easy.

We collect some properties of the operators $G(T)$.

Lemma 3.6. Let $S, T \in \mathcal{E}_{0}^{\prime}, \varphi \in \mathcal{D}_{0}, \psi \in \mathcal{D}$ and $x \in E$. Then

a) for all $m \in \mathbb{N}:(G(\varphi) x, G(\underbrace{T * \ldots * T}_{m} * \varphi) x) \in G(T)^{m}$.

b) $G(\psi)$ commutes with $G(T)$.

c) $G(S) G(T) \subset G(S * T)$ with $D(G(S) G(T))=D(G(S * T)) \cap D(G(T))$, and $G(S)+G(T) \subset G(S+T)$.

d) $\left(G(\psi) x, G\left(-\psi^{\prime}\right) x-\psi(0) x\right) \in A$.

Proof. Let $\tilde{\varphi} \in \mathcal{D}_{0}$. Using (3) we get

$$
G(T * \tilde{\varphi}) G(\varphi) x=G(T * \tilde{\varphi} * \varphi) x=G(\tilde{\varphi}) G(T * \varphi) x
$$

which proves a) for $m=1$. The rest is induction. Using (3) and the definition of $G(T)$ we get

$$
G(T * \tilde{\varphi}) G(\psi) x=G(\psi) G(T * \tilde{\varphi}) x=G(\psi) G(\tilde{\varphi}) G(T) x=G(\tilde{\varphi}) G(\psi) G(T) x
$$

which proves b). If $x \in D(G(S) G(T))$, then

$$
G((T * S) * \tilde{\varphi}) x=G(T *(S * \tilde{\varphi})) x=G(S * \tilde{\varphi}) G(T) x=G(\tilde{\varphi}) G(S) G(T) x,
$$

and if $(x, z) \in G(S * T),(x, y) \in G(T)$, then by the definition of $G(T)$ and $G(S * T)$

$$
G(S * \tilde{\varphi}) y=G(T *(S * \tilde{\varphi})) x=G((S * T) * \tilde{\varphi}) x=G(\tilde{\varphi}) z,
$$


from which we first get $(y, z) \in G(S)$ and then $(x, z) \in G(S) G(T)$. If $x \in D(G(S)) \cap$ $D(G(T))$, then

$$
G((S+T) * \tilde{\varphi}) x=G(S * \tilde{\varphi}) x+G(T * \tilde{\varphi}) x=G(\tilde{\varphi})(G(S) x+G(T) x),
$$

so $\mathrm{c}$ ) is proved. Using (3) and (1) we get

$$
\begin{aligned}
G\left(-\delta^{\prime} * \tilde{\varphi}\right) G(\psi) x & =G\left(-\tilde{\varphi}^{\prime}\right) G(\psi) x=G\left(-\tilde{\varphi}^{\prime} *_{0} \psi\right) x \\
& =G\left(\tilde{\varphi} *_{0}\left(-\psi^{\prime}\right)-\psi(0) \tilde{\varphi}\right) x \\
& =G(\tilde{\varphi})\left[G\left(-\psi^{\prime}\right) x-\psi(0) x\right]
\end{aligned}
$$

which proves d).

Corollary 3.7. If the DSG $G$ is dense its generator is densely defined.

The following example shows that the inclusion in c) is proper in general.

Example 3.8. Let the DSG $G$ be induced by a $C_{0}$-SG with unbounded generator $A$. Let $S:=\varphi \in \mathcal{D}_{0}$ and $T:=-\delta^{\prime}$. Because of $D(A) \neq E$ we have

$$
G(S) G(T)=G(\varphi) A \neq G\left(-\varphi^{\prime}\right)=G(T * S) .
$$

We will need the following definition.

Definition 3.9. Let $D$ be another Banach space and $P \in \mathcal{D}_{0}^{\prime}(L(D, E))$. A fundamental solution for $P$ is an element $G$ of $\mathcal{D}_{0}^{\prime}(L(E, D))$ satisfying

$$
P * G=\delta \otimes I d_{E} \quad \text { and } \quad G * P=\delta \otimes I d_{D} .
$$

Since the convolution is associative, fundamental solutions are unique. The importance of fundamental solutions is illustrated by the fact that if $P$ has a fundamental solution $G$, then for each $T \in \mathcal{D}_{0}^{\prime}(E)$ the equation $P * U=T$ has a unique solution $U \in \mathcal{D}_{0}^{\prime}(D)$ which is given by $U:=G * T$. The following theorem is therefore the central key for studying Cauchy problems and justifies our definitions.

Theorem 3.10. Let $A$ be a closed operator in $E$. Then $A$ generates a $D S G$ if and only if there is a fundamental solution for $P:=\delta^{\prime} \otimes I-\delta \otimes A \in \mathcal{D}_{0}^{\prime}(L(D(A), E))$ where $D(A)$ is supplied with the graph norm and I denotes the inclusion $D(A) \rightarrow E$.

Proof. If $A$ generates the DSG $G$, then by Lemma 3.6 d) $G$ is a fundamental solution for $P$. So let $G$ be a fundamental solution for $P$. We will show that $G$ is a DSG with generator $A$.

To show (3) let $\psi \in \mathcal{D}$ and $x \in E$. If $U:=G(\cdot) G(\psi) x$ and $V:=G\left(\cdot *_{0} \psi\right) x$, then $U, V \in \mathcal{D}_{0}^{\prime}(D(A))$ and $P * U=\delta \otimes G(\psi) x=P * V$. Since $G$ is a fundamental solution for $P$ and the convolution is associative we obtain $U=V$ which implies (3).

To show (4) we proceed as in [16]. Let $x \in \mathcal{N}(G)$. Then the support of the distribution $G(\cdot) x$ is contained in $\{0\}$. So (see [14], Lemma 8.1.1) there exist a $k \in \mathbb{N}_{0}$ and elements $y_{0}, \ldots, y_{k} \in D(A)$ such that

$$
G(\cdot) x=\sum_{j=0}^{k} \delta^{(j)} \otimes y_{j} .
$$

Since $G$ is a fundamental solution we obtain

$$
\sum_{j=0}^{k} \delta^{(j+1)} \otimes y_{j}-\sum_{j=0}^{k} \delta^{(j)} \otimes A y_{j}=\delta \otimes x
$$


which implies $y_{k}=0$. By induction we get $y_{j}=0$ for all $j \in\{0, \ldots, k\}$ which implies $x=0$.

Let $\tilde{A}$ denote the generator of $G$. If $(x, y) \in A$, then

$$
G\left(-\varphi^{\prime}\right) x-G(\varphi) y=0
$$

for all $\varphi \in \mathcal{D}_{0}$ since $G$ is a fundamental solution. So $(x, y) \in \tilde{A}$, and consequently $A \subset \tilde{A}$. This implies that $\mathcal{D}_{0}^{\prime}(D(A))$ is an isomorphic subspace of $\mathcal{D}_{0}^{\prime}(D(\tilde{A}))$. Applying the first part of the theorem, we see that $G$ is a fundamental solution for

$$
\tilde{P}:=\delta^{\prime} \otimes \tilde{I}-\delta \otimes \tilde{A} .
$$

So $G *$ is an isomorphism from $\mathcal{D}_{0}^{\prime}(E)$ onto $\mathcal{D}_{0}^{\prime}(D(A))$ and onto $\mathcal{D}_{0}^{\prime}(D(\tilde{A}))$ which implies $\mathcal{D}_{0}^{\prime}(D(A))=\mathcal{D}_{0}^{\prime}(D(\tilde{A}))$ and hence $D(A)=D(\tilde{A})$.

In what follows, we will call a fundamental solution for $\delta^{\prime} \otimes I-\delta \otimes A$ simply a fundamental solution for $A$.

Proposition 3.11. Let $G$ be a DSG with generator $A$. Then the following assertions are equivalent:

a) $G$ is a dense $D S G$.

b) $G(\cdot)^{*}$ is a $D S G$ in $E^{*}$.

c) $A$ is densely defined.

Proof. Assume c). By Theorem $3.10 G$ is a fundamental solution for $A$. But then $G(\cdot)^{*}$ is a fundamental solution for the closed operator $A^{*}$ in $E^{*}$. Applying Theorem 3.10 once more we get that $G(\cdot)^{*}$ is a DSG in $E^{*}$ with generator $A^{*}$. So b) holds. If b) holds we get from Lemma 2.7 and the theorem of bipolars

$$
E=\left\{0_{E^{*}}\right\}^{\circ}=\left(\mathcal{N}\left(G(\cdot)^{*}\right)\right)^{\circ}=\overline{\mathcal{R}(G)}^{\circ \circ}=\overline{\mathcal{R}(G)},
$$

so $G$ is dense, and a) holds. By Corollary 3.7 , a) implies c).

Using Theorem 1.6 in [6] or Theorem 2.5 in [12] we get

Corollary 3.12. Let $A$ be a closed operator in E. A is the generator of a DSG if and only if there are constants $\alpha, \beta, C>0$ and $n \in \mathbb{N}_{0}$ such that

$$
\Lambda:=\{\lambda \in \mathbb{C}: \operatorname{Re} \lambda \geq \alpha \log (1+|\lambda|)+\beta\} \subset \rho(A)
$$

and

$$
\forall \lambda \in \Lambda:\|R(\lambda, A)\| \leq C(1+|\lambda|)^{n} .
$$

For the proof we only have to note that by the resolvent equation $\|R(\cdot, A)\|_{L(E)}$ is polynomially bounded on $\Lambda$ if and only if $\|R(\cdot, A)\|_{L(E, D(A))}$ is polynomially bounded on $\Lambda$.

Remark 3.13. In [16] a distribution semigroup was defined to be an element $G$ in $\mathcal{D}_{0}^{\prime}(L(E))$ satisfying

$$
\forall \varphi, \psi \in \mathcal{D}_{0}: G(\varphi) G(\psi)=G(\varphi * \psi)
$$

the conditions (4), (5) and in addition

(8)

$$
\forall \varphi \in \mathcal{D}_{0}, x \in E \exists u \in C\left(\left[0, \infty[, E) \forall \psi \in \mathcal{D}: G(\psi) G(\varphi) x=\int_{0}^{\infty} \psi(t) u(t) d t .\right.\right.
$$

The definition in [16] requires for the $u$ in (8) that $u(0)=G(\varphi) x$, but (7) implies that $u(t)=G\left(\tau_{t} \varphi\right) x$ for $t>0$, and so this condition is automatically fulfilled by 
the continuity of $u$. We will call a $G$ satisfying (7), (4), (5) and (8) a $D S G$ - $L$ for the moment. The generator of a DSG-L $G$ was in [16] defined to be the closure of the closable linear operator

(9)

$$
\begin{aligned}
& \left\{(x, y): \exists \text { regularizing sequence }\left(\rho_{n}\right) \text { in } \mathcal{D}_{0}:\right. \\
& \left.\qquad \lim _{n} G\left(\rho_{n}\right) x=x \text { and } \lim _{n} G\left(-\rho_{n}^{\prime}\right) x=y\right\} .
\end{aligned}
$$

It was then shown in [16] that the assertion of Theorem 3.10 holds for densely defined closed linear operators $A$ with "DSG" replaced by "DSG-L". Now being a DSG-L is equivalent to being a dense DSG. Indeed, clearly (3) implies (7) and moreover

$$
G(\psi) G(\varphi) x=G\left(\psi *_{0} \varphi\right) x=G\left(\int_{0}^{\infty} \psi(t) \tau_{t} \varphi d t\right) x=\int_{0}^{\infty} \psi(t) G\left(\tau_{t} \varphi\right) x d t
$$

for all $\psi \in \mathcal{D}, \varphi \in \mathcal{D}_{0}$ and $x \in E$, which in turn implies (8). On the other hand, if $G$ is a DSG-L, then Lemmas 3.2 and 2.1 in [16] imply

$$
\begin{aligned}
G\left(\psi_{1}\right) G\left(\psi_{2}\right) G(\varphi) & =G\left(\left(\psi_{1} 1_{[0, \infty[}\right) *\left(\psi_{2} 1_{[0, \infty[}\right) * \varphi\right)=G\left(\left(\left(\psi_{1} *_{0} \psi_{2}\right) 1_{[0, \infty[}\right) * \varphi\right) \\
& =G\left(\psi_{1} *_{0} \psi_{2}\right) G(\varphi)
\end{aligned}
$$

for all $\psi_{1}, \psi_{2} \in \mathcal{D}$ and $\varphi \in \mathcal{D}_{0}$. Hence it follows from (5) that (3) holds.

So it is clear that our definition of the generator coincides with the one given in [16] for the case of dense DSGs. It does not coincide for non-dense DSGs. In fact it may be shown that for a non-dense DSG $G$ with generator $A$ the operator given by (9) coincides with the closure of the restriction of $A$ to $D_{\infty}(A):=\bigcap_{n} D\left(A^{n}\right)$.

We want to point out that our definition of a DSG cannot be obtained by simply dropping the denseness assumption (5) from the definition of a DSG-L. Indeed, let $G$ be a non-dense DSG with generator $A$. Then $D(A)$ is not dense in $E$, so there are $x \in E$ and $x^{*} \in(D(A))^{\circ}$ with $\left\langle x^{*}, x\right\rangle \neq 0$. Then $\tilde{G}:=G+\delta \otimes\left\langle x^{*}, \cdot\right\rangle x$ satisfies (7), (4), (8), but not (3).

Moreover, our definition has the advantage of being more algebraic and avoiding the somewhat crude assumption (8).

\section{RePresentations of Distribution SEMigRoups, LOCAL INTEGRATED SEMIGROUPS}

It is well known that each distribution is locally the iterated derivative of a continuous function. We recall the following fact (see for instance [14], Theorem 8.1.5).

Proposition 4.1. Let $F$ be a Banach space, $a>0$ and $n \in \mathbb{N}_{0}$. Suppose $G \in$ $\mathcal{D}_{0}^{\prime}(F)$ has a continuous extension to the space

$$
D_{1}^{n}[0, a]:=\left(\left\{f \in C^{n}([0, a], F): \forall j \leq n: f^{(j)}(a)=0\right\},\|\cdot\|_{D_{1}^{n}}\right)^{\sim}
$$

where $\|f\|_{D_{1}^{n}}:=\sum_{j=0}^{n}\left\|f^{(j)}\right\|_{L^{1}}$. Then

$$
G(\psi)=\int_{0}^{a}(-1)^{n+1} \psi^{(n+1)}(t) g(t) d t
$$

for all $\psi \in \mathcal{D}$ with support contained in $]-\infty, a]$ where $g:[0, a] \rightarrow F$ is the continuous function

and $I_{t}^{n+1}(s):=\left((t-s)^{n} / n !\right) 1_{[0, t]}(s)$.

$$
t \mapsto g(t):=G\left(I_{t}^{n+1}\right),
$$


Remark 4.2. Note that $\left(I_{t}^{n+1}\right)^{\prime}=-I_{t}^{n}$ and that $(d / d t) I_{t}^{n+1}=I_{t}^{n}$ if we set $I_{t}^{0}:=\delta_{t}$.

We call a function $g$ satisfying (10) a continuous representation of order $n+$ 1 on $[0, a]$ of $G$. If $F=L(E)$ it may happen that $g$ is strongly differentiable in which case we call $g^{\prime}$ a strongly continuous representation of $G$. Notice that (strongly) continuous representations in this sense are unique. In the following we are interested in strongly continuous representations of DSGs or even pre-DSGs. We start with

Proposition 4.3. Let $G$ be a pre-DSG in $E, a>0$ and $n \in \mathbb{N}$. Suppose $G$ has a strongly continuous representation $F$ of order $n$ on $[0, a]$. Then

a) For all $s, t \in[0, a]$ with $s+t \in[0, a]$,

$$
F(t) F(s)=\int_{t}^{s+t} \frac{(s+t-r)^{n-1}}{(n-1) !} F(r) d r-\int_{0}^{s} \frac{(s+t-r)^{n-1}}{(n-1) !} F(r) d r .
$$

Furthermore

$$
\operatorname{Kern} T=\bigcap_{t \in] 0, a]} \operatorname{Kern} F(t) \quad \text { and } \quad \operatorname{Kern} S=\left(\overline{\bigcup_{t \in] 0, a]} \operatorname{Im} F(t)}\right)^{\circ},
$$

where $T \in L(\mathcal{N}(G))$ denotes the kernel operator of $G$ and $S$ denotes the kernel operator of $G(\cdot)^{*}$ (see Lemma 2.2).

b) If $G$ is a DSG with generator A, then

$$
(x, y) \in A \Leftrightarrow \forall t \in[0, a]: F(t) x-\frac{t^{n}}{n !} x=\int_{0}^{t} F(s) y d s .
$$

The proof is based on the following lemma.

Lemma 4.4. For all $n \in \mathbb{N}_{0}$ and $s, t \geq 0$,

$$
I_{t}^{n} * I_{s}^{n}=I_{s+t}^{2 n}-\sum_{j=0}^{n-1}\left(\frac{s^{j}}{j !} I_{t}^{2 n-j}+\frac{t^{j}}{j !} I_{s}^{2 n-j}\right) .
$$

Proof. For $n=0$ the assertion is clear. For $n=1$ and $r \geq 0$ we have

$$
I_{t}^{1} * I_{s}^{1}(r)=1_{[0, t]} * 1_{[0, s]}(r)=\int_{0}^{r} 1_{[0, t]}(\rho) 1_{[0, s]}(r-\rho) d \rho=\int_{0}^{r} 1_{[0, t] \cap[r-s, r]}(\rho) d \rho .
$$

For $s \geq t$ this equals

$$
\begin{aligned}
& r 1_{[0, t]}(r)+t 1_{[t, s]}+(s+t-r) 1_{[s, s+t]}(r) \\
& \quad=(s+t-r) 1_{[0, s+t]}(r)-(s-r) 1_{[0, s]}(r)-(t-r) 1_{[0, t]}(r) .
\end{aligned}
$$

So the assertion holds for $n=1$. If it holds for $n \in \mathbb{N}$, we have

$$
\begin{aligned}
\left(I_{t}^{n+1} * I_{s}^{n+1}\right)^{\prime \prime} & =I_{t}^{n} * I_{s}^{n}-\left(I_{s}^{n+1}(0) I_{t}^{n}+I_{t}^{n+1}(0) I_{s}^{n}\right) \\
& =I_{s+t}^{2 n}-\sum_{j=0}^{n}\left(\frac{s^{j}}{j !} I_{t}^{2 n-j}+\frac{t^{j}}{j !} I_{s}^{2 n-j}\right) .
\end{aligned}
$$

Integrating twice we get the assertion for $n+1$. 
Proof of Proposition 4.3. Notice first that we have

$$
F(t) x=\frac{d}{d t} G\left(I_{t}^{n}\right) x
$$

for all $x \in E$ and $t \in[0, a]$. We start with a), so let $x \in E$ and $s, t \geq 0$ with $s+t \leq a$. Using (3), Lemma 4.4 and Remark 4.2 we get

$$
\begin{aligned}
G\left(I_{t}^{n+1}\right) G\left(I_{s}^{n+1}\right) x & =G\left(I_{t}^{n+1} * I_{s}^{n+1}\right) x \\
& =G\left(I_{s+t}^{2 n+2}-\sum_{j=0}^{n}\left(\frac{s^{j}}{j !} I_{t}^{2 n+2-j}+\frac{t^{j}}{j !} I_{s}^{2 n+2-j}\right)\right) \\
& =G\left(I_{s+t}^{2 n+2}\right)-\sum_{j=0}^{n}\left(\frac{s^{j}}{j !} G\left(I_{t}^{2 n+2-j}\right)+\frac{t^{j}}{j !} G\left(I_{s}^{2 n+2-j}\right)\right) \\
& =\int_{s}^{t+s} \frac{(s+t-r)^{n}}{n !} G\left(I_{r}^{n+1}\right) d r-\int_{0}^{s} \frac{(s+t-r)^{n}}{n !} G\left(I_{r}^{n+1}\right) d r .
\end{aligned}
$$

Differentiating this equation with respect to $s$ and once more with respect to $t$ we obtain (11).

To show the characterization of $\operatorname{Kern} T$ let $x \in \mathcal{N}(G)$. Then there are $y_{0}, \ldots$, $y_{n-1} \in E$ with $F(t) x=\sum_{j=0}^{n-1} y_{j} t^{j} / j$ ! for $\left.\left.t \in\right] 0, a\right]$. If $\psi \in \mathcal{D}$ is such that $\psi=1$ on a neighbourhood of 0 , then integrating by parts $n$ times gives

$$
T x=G(\psi) x=(-1)^{n} \int_{0}^{a} \psi^{(n)}(t) F(t) x=\left.\psi(0)\left(\left(\frac{d}{d t}\right)^{n-1} F(t) x\right)\right|_{t=0}=y_{n-1} .
$$

For $x \notin \operatorname{Kern} T$ this implies $x \notin \bigcap \operatorname{Kern} F(t)$. For $x \in \operatorname{Kern} T$ we get by Lemma

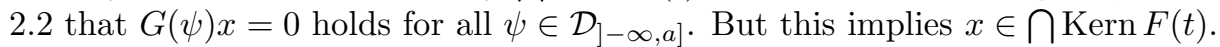

Now we get the remaining equality by dualization. If $t \mapsto F(t)^{*}$ is not strongly continuous, then $t \mapsto F_{1}(t):=\int_{0}^{t} F(s) d s$ is a norm-continuous representation of $G$ of order $n+1$ and $t \mapsto F_{1}(t)^{*}$ is a norm-continuous representation of $G(\cdot)^{*}$ of order $n+1$. Hence

$$
\operatorname{Kern} S=\bigcap \operatorname{Kern} F_{1}(t)^{*}=\left(\overline{\left(\operatorname{Im} F_{1}(t)\right.}\right)^{\circ}=(\overline{\bigcup \operatorname{Im} F(t)})^{\circ}
$$

by the equality already shown, and a) is proved.

To show b) let $x, y \in E$. Suppose first that

$$
\forall t \in[0, a]: F(t) x-\frac{t^{n}}{n !} x=\int_{0}^{t} F(s) y d s
$$

and let $\varphi \in \mathcal{D}_{[0, a]}$. Then integration by parts gives

$$
\begin{aligned}
G\left(-\varphi^{\prime}\right) x & =\int_{0}^{a}(-1)^{n+1} \varphi^{(n+1)}(t)\left(F(t) x-\frac{t^{n}}{n !} x\right) d t \\
& =\int_{0}^{a}(-1)^{n} \varphi^{(n)}(t) F(t) y d t=G(\varphi) y
\end{aligned}
$$

which implies $(x, y) \in A$. Assume now that $(x, y) \in A$. Since $G$ is a fundamental solution for $A$ integration by parts gives for all $\psi \in \mathcal{D}_{]-\infty, a]}$

$$
\int_{0}^{a}(-1)^{n+1} \psi^{(n+1)}(t)\left(F(t) x-\int_{0}^{t} F(s) y d s\right) d t=\psi(0) x .
$$


Hence

$$
\forall t \in[0, a]:(-1)^{(n+1)}\left(F(t) x-\int_{0}^{t} F(s) y d s\right)=(-1)^{n+1} x \frac{t^{n}}{n !}
$$

which finishes the proof of $b$ ).

Corollary 4.5. Let the assumptions of Proposition 4.3 hold. Then

a) $G$ is a DSG if and only if $\bigcap_{t \in] 0, a]} \operatorname{Kern} F(t)=\{0\}$.

b) $G$ is a dense pre-DSG if and only if $\overline{\bigcup_{t \in] 0, a]} \operatorname{Im} F(t)}=E$.

Proof. b) follows from a) by dualization and Lemma 2.7, so let us show a). If $G$ is a DSG, then $\operatorname{Kern} T=\{0\}$. If $G$ is not a DSG, then $\mathcal{N}(G) \neq\{0\}$. Hence Kern $T \neq\{0\}$ since $T$ is nilpotent (see Lemma 2.2), so we are finished by Proposition $4.3 \mathrm{a})$.

If $G$ is a DSG with generator $A$ we also get operators $G\left(I_{t}^{n}\right)$ according to Definition 3.1. Notice that these will coincide with the operators obtained by continuous extension (see Proposition 4.1) in case the latter are defined.

Proposition 4.6. Let $G$ be a DSG with generator $A, x \in E$ and $n \in \mathbb{N}_{0}$. Then the following assertions are equivalent.

a) There is a $u \in C([0, a], E)$ such that for all $\psi \in \mathcal{D}_{]-\infty, a]}$,

$$
G(\psi) x=\int_{0}^{a}(-1)^{n} \psi^{(n)}(t) u(t) d t
$$

b) $x \in \bigcap_{t \in[0, a]} D\left(G\left(I_{t}^{n}\right)\right)$ and $t \mapsto G\left(I_{t}^{n}\right) x \in C([0, a], E)$.

c) There is a $v \in C([0, a], E)$ such that for all $t \in[0, a]$,

$$
\left(\int_{0}^{t} v(s) d s, v(t)-\left(t^{n} / n !\right) x\right) \in A \text {. }
$$

Then $u=v=G\left(I^{n}\right) x$.

Proof. a) $\rightarrow$ b): Let a) hold and $\varphi \in \mathcal{D}_{0}$. Then for all $\psi \in \mathcal{D}_{]-\infty, a]}$,

$$
\begin{aligned}
\int_{0}^{a}(-1)^{n} \psi^{(n)}(t) G\left(I_{t}^{n} * \varphi\right) x d t & =G\left(\int_{0}^{a}(-1)^{n} \psi^{(n)}(t) \varphi * I_{t}^{n} d t\right) x \\
& =G\left(\psi *_{0} \varphi\right) x \quad(n \text { times integration by parts) } \\
& =G(\varphi) G(\psi) x \\
& =\int_{0}^{a}(-1)^{n} \psi^{(n)}(t) G(\varphi) u(t) d t
\end{aligned}
$$

Hence $G\left(I_{t}^{n} * \varphi\right) x=G(\varphi) u(t)$ for all $t \in[0, a]$. Since $\varphi \in \mathcal{D}_{0}$ was arbitrary and $u$ is continuous, b) holds.

b) $\rightarrow$ c): Suppose b) holds. Let $\varphi \in \mathcal{D}_{0}$ and $t \in[0, a]$. Then

$$
G\left(-\varphi^{\prime}\right) \int_{0}^{t} G\left(I_{s}^{n}\right) x d s=\int_{0}^{t} G\left(-\varphi^{\prime} * I_{s}^{n}\right) d s x=G\left(-\int_{0}^{t} \varphi^{\prime} * I_{s}^{n} d s\right) x
$$

Since $\varphi \in \mathcal{D}_{0}$ we have by Remark 4.2 and (1)

$$
\begin{aligned}
-\int_{0}^{t} \varphi^{\prime} * I_{s}^{n} d s & =-\varphi^{\prime} * \int_{0}^{t} I_{s}^{n} d s=-\varphi^{\prime} * I_{t}^{n+1} \\
& =\varphi *\left(-I_{t}^{n+1}\right)^{\prime}-I_{t}^{n+1}(0) \varphi=\varphi * I_{t}^{n}-\frac{t^{n}}{n !} \varphi
\end{aligned}
$$


Thus

$$
G\left(-\varphi^{\prime}\right) \int_{0}^{t} G\left(I_{s}^{n}\right) x d s=G(\varphi)\left(G\left(I_{t}^{n}\right) x-\frac{t^{n}}{n !} x\right),
$$

and hence c) follows by putting $v:=G\left(I^{n}\right) x$.

c) $\rightarrow$ a): Suppose c) holds. Let $V(\psi):=\int_{0}^{a}(-1)^{n} \psi^{(n)}(t) v(t) d t$ for all $\psi \in \mathcal{D}_{]-\infty, a]}$. Then for those $\psi$ integrating by parts yields

$$
V(\psi)=\int_{0}^{a}(-1)^{n+1} \psi^{(n+1)}(t) \int_{0}^{t} v(s) d s d t,
$$

from which we get $V(\psi) \in D(A)$ and

$$
A V(\psi)=V\left(-\psi^{\prime}\right)-\int_{0}^{a}(-1)^{n+1} \psi^{(n+1)}(t) \frac{t^{n}}{n !} d t x=V\left(-\psi^{\prime}\right)-\psi(0) x .
$$

Since $A$ is the generator of $G$ this implies $V(\psi)=G(\psi) x$ for all $\psi \in \mathcal{D}_{]-\infty, a]}$. So we get a) by putting $u:=v$.

The following proposition will help to get mild solutions of the abstract Cauchy problem.

Proposition 4.7. Let $G$ be a DSG with generator $A$ and $n \in \mathbb{N}, t>0$. Suppose $x \in D(A)$ with $A x \in D\left(G\left(I_{t}^{n}\right)\right)$. Then $\left(x, G\left(I_{t}^{n}\right) A x+\left(t^{n-1} /(n-1) !\right) x\right) \in G\left(I_{t}^{n-1}\right)$.

Proof. Let $\varphi \in \mathcal{D}_{0}$. Then

$$
\begin{aligned}
G(\varphi)\left(G\left(I_{t}^{n}\right) A x+\frac{t^{n-1}}{(n-1) !} x\right) & =G\left(\varphi * I_{t}^{n}\right) A x+G\left(\frac{t^{n-1}}{(n-1) !} \varphi\right) x \\
& =G\left(-\left(\varphi * I_{t}^{n}\right)^{\prime}\right) x+G\left(\frac{t^{n-1}}{(n-1) !} \varphi\right) x \\
& =G\left(\varphi *\left(-\left(I_{t}^{n}\right)^{\prime}\right)\right) x=G(\varphi) G\left(I_{t}^{n-1}\right) x .
\end{aligned}
$$

Using Proposition 4.1 we get from Propositions 4.6 and 4.7.

Corollary 4.8. Let $G$ be a $D S G$ with generator $A$ and $a>0$. Then there is an $n \in \mathbb{N}_{0}$ such that

$$
\left\{\begin{array}{l}
\text { for all } x \in E \text { the equation } \forall t \in[0, a]: A \int_{0}^{t} v(s) d s=v(t)-\left(t^{n} / n !\right) x \\
\text { has a unique solution } v=v(\cdot, x) \in C([0, a], E) .
\end{array}\right.
$$

If $n \in \mathbb{N}_{0}$ is such that (13) holds then

$$
\left\{\begin{array}{l}
\text { for all } x \in D\left(A^{n}\right) \text { the equation } \forall t \in[0, a]: A \int_{0}^{t} v(s) d s=v(t)-x \\
\text { has a unique solution } v=v(\cdot, x) \in C([0, a], E) .
\end{array}\right.
$$

Definition 4.9. Following [15] and [2] we will call a closed linear operator $A$ in $E$ satisfying (13) the generator of the local n-times integrated semigroup $S: t \mapsto$ $v(t, \cdot)$ on $[0, a]$. Note that by the closed graph theorem the closed linear mapping $E \rightarrow C([0, a], E), x \rightarrow v(\cdot, x)$ is continuous, so $S$ defines a mapping with values in $L(E)$ which is strongly continuous. 
Remark 4.10. In [23] local $n$-times integrated semigroups on $[0, a]$ are defined as strongly continuous applications $F:[0, a] \rightarrow L(E)$ satisfying equation (11) and $\bigcap_{t} \operatorname{Kern} F(t)=\{0\}$. This definition is equivalent to Definition 4.9 if we define the generator $A$ of $F$ by (12). The definition of the generator in [23] is adapted to the case that $\bigcup_{t} \operatorname{Im} F(t)$ is dense in $E$ and is in general not equivalent to the definition in [2], but it is if $A$ is densely defined.

For the case of (local) integrated semigroups on $[0, \infty[,(12)$ is used as a definition for the generator in [24] (where $n=1$ ) and [18].

The definitions used for exponentially bounded $n$-times integrated semigroups (see [1]) involve Laplace transforms and cannot be used in the local situation.

The following theorem was proved in [23] and in [15], [2] for the case that $A$ is densely defined.

Theorem 4.11. Let $A$ be a closed linear operator in E. Then the following assertions are equivalent:

a) A generates a DSG.

b) For all $a>0$ there is an $n \in \mathbb{N}_{0}$ such that $A$ generates a local n-times integrated semigroup on $[0, a]$.

c) There are $a>0$ and $n \in \mathbb{N}_{0}$ such that $A$ generates a local $n$-times integrated semigroup on $[0, a]$.

Proof. The implication $a) \rightarrow b$ ) is Corollary 4.8 and the implication $b) \rightarrow c$ ) is trivial. So assume c) holds. Let $S$ denote the $n$-times integrated semigroup generated by $A$ on $[0, a]$. Let $G_{0}$ be defined by $G_{0}(\psi) x:=\int_{0}^{a}(-1)^{n} \psi^{(n)}(t) S_{t} x d t$. Then $G_{0} \in \mathcal{D}_{0}^{\prime}(L(E, D(A)))$ and integration by parts gives

$$
\begin{aligned}
& \left(G_{0}^{\prime}-A G_{0}\right)(\psi) x \\
= & \int_{0}^{a}(-1)^{n+1} \psi^{(n+1)}(t)\left(S_{t} x-A \int_{0}^{s} S_{s} x d s\right) d t-(-1)^{n} \psi^{(n)}(a) A \int_{0}^{a} S_{s} x d s \\
= & \int_{0}^{a}(-1)^{n+1} \psi^{(n+1)}(t) \frac{t^{n}}{n !} d t x-(-1)^{n} \psi^{(n)}(a)\left(S_{a} x-\frac{a^{n}}{n !} x\right) \\
= & \psi(0) x-\left(\sum_{j=0}^{n-1}(-1)^{j} \psi^{(j)}(a) \frac{t^{j}}{j !}\right) x-(-1)^{n} \psi^{(n)}(a) S_{a} x \\
= & \left(\delta \otimes I d_{E}-H\right)(\psi) x
\end{aligned}
$$

where supp $H \subset\{a\}$. For $x \in D(A)$ we have $A G_{0}(\cdot) x=G_{0}(\cdot) A x$, so if $K:=$ $\left.H(\cdot)\right|_{D(A)}$, then

$$
G_{0} * P=G_{0}^{\prime}-G_{0} A=\delta \otimes I d_{D(A)}-K .
$$

So a) follows from Theorem 3.10 and the following lemma which is formulated for convolution equations in the sense of [12] and [13].

Lemma 4.12. Let $X$ and $Y$ be Banach spaces, $a>0$, and $P \in \mathcal{D}_{0}^{\prime}(L(Y, X))$, $G_{0} \in \mathcal{D}_{0}^{\prime}(L(X, Y))$ be such that

$$
P * G_{0}=I-H \quad \text { and } \quad G_{0} * P=J-K
$$


where $I:=\delta \otimes I d_{X}, J:=\delta \otimes I d_{Y}$ and $\operatorname{supp} H \cup \operatorname{supp} K \subset[a, \infty[$. Then $P$ has a fundamental solution $G$ given by

$$
G:=G_{0} *\left(\sum_{j=0}^{\infty} H^{* j}\right)
$$

where $H^{* 0}:=I$ and $H^{* j}$ denotes the $j$-th convolution power of $H$ for $j \geq 1$.

Proof. Note first that $\operatorname{supp} H^{* j} \subset[j a, \infty$ [ for each $j$, and so the series in (16) is locally finite and defines an element in $\mathcal{D}_{0}^{\prime}(L(X))$ which will be denoted by $V$. From (15) we get

$$
P-P * K=P *(J-K)=P * G_{0} * P=(I-H) * P=P-H * P,
$$

i.e. $H * P=P * K$. If we set $W:=\sum_{j=0}^{\infty} K^{* j}$ where $K^{* 0}:=J$ this implies $V * P=P * W$ since the convolution is continuous. Now

$$
P * G=P * G_{0} * V=(I-H) * V=I
$$

and

$$
G * P=G_{0} * V * P=G_{0} * P * W=(J-K) * W=J,
$$

and the lemma is proved.

\section{Distribution SEMigroups AND REgularized SEMigroups}

We will use the following definition from [10].

Definition 5.1. A regularized semigroup is a strongly continuous mapping $S$ : $[0, \infty[\rightarrow L(E)$ with

$$
\text { ker } S_{0}=\{0\} \quad \text { and } \quad \forall s, t \geq 0: S_{t} S_{s}=S_{s+t} S_{0} .
$$

The generator of $S$ is then given by

$$
\left\{(x, y): \lim _{t \rightarrow 0} \frac{1}{t}\left(S_{t} x-S_{0} x\right)=S_{0} y\right\}
$$

and is a closed linear operator in $E$. A given closed linear operator $A$ is said to generate a $C$-regularized semigroup where $C \in L(E)$ is injective if there is a regularized semigroup $S$ with $S_{0}=C$ whose generator is $A$.

Let now $A$ be the generator of a DSG $G$ in $E$. We will show that there is an injective $C$ such that $A$ generates a $C$-regularized semigroup. For this purpose we recall the following which was proved in [9], Theorem 1.5 (see also [22], Corollary 2.2 , and [10], Theorem 4.15).

Proposition 5.2. Suppose $B$ is a closed linear operator in $E$ with $\rho(B) \neq \emptyset$ and $C \in L(B)$ is injective and commutes with $B$. Suppose further that for all $x \in \operatorname{Im} C$ the Cauchy problem

$$
u^{\prime}(t)=B u(t), t \geq 0, \quad u(0)=x
$$

has a unique mild solution. Then $B$ generates a $C$-regularized semigroup.

Now by Corollary 3.12 there are constants $\alpha, \beta, C>0$ and $k \in \mathbb{N}_{0}$ such that

$$
\Lambda:=\{\xi+i \eta: \xi \geq \alpha \log (1+|\eta|)+\beta\} \subset \rho(A)
$$

and all $\lambda \in \Lambda$ satisfy

$$
\|R(\lambda, A)\| \leq C(1+|\lambda|)^{k} .
$$


Passing from $A$ and $G$ to $A-(\beta+1)$ and $e^{-(\beta+1)(\cdot)} G$, respectively, we may assume $\beta \leq-1$ without loss of generality, since $A-\mu$ generates the $C$-RSG $\left(e^{-\mu t} S_{t}\right)$ if $A$ generates the $C$-RSG $\left(S_{t}\right)$.

We denote by $\Gamma$ the boundary of $\Lambda$, i.e.

$$
\Gamma:=\{\xi+i \eta: \xi=\alpha \log (1+|\eta|)+\beta\},
$$

parameterized by $\eta$, i.e. by

$$
\gamma: \mathbb{R} \rightarrow \mathbb{C}, \gamma(\eta):=\alpha \log (1+|\eta|)+\beta+i \eta
$$

Let Log denote the branch of the logarithm that is holomorphic on $\mathbb{C} \backslash]-\infty, 0]$ satisfying $\log 1=0$, and let $(\cdot)^{b}:=\exp \circ(b \log )$ and

$$
S_{b}:=\left\{z \in \mathbb{C} \backslash\{0\}:|\arg z|<\frac{\pi(1-b)}{4}\right\}
$$

for all $b \in] 0,1[$. We now fix $b \in] 0,1[$ and define

$$
C_{b, \tau}:=\exp \left(-\tau(-A)^{b}\right):=\frac{1}{2 \pi i} \int_{\Gamma} \exp \left(-\tau(-\lambda)^{b}\right) R(\lambda, A) d \lambda
$$

for all $\tau \in S_{b}$. We will now show that these operators have the desired properties (see also [10], Example 22.31). We start with

Lemma 5.3. Let $\tau \in S_{b}$. Then $C_{b, \tau} \in L(E), C_{b, \tau}$ commutes with $A$ and $\operatorname{Im} C_{b, \tau} \subset$ $D_{\infty}(A)$.

Proof. Denote by $\Omega$ the complement of $\Lambda$ in $\mathbb{C}$. Denote by arg the argument on $\mathbb{C} \backslash]-\infty, 0]$ with values in $]-\pi, \pi\left[\right.$. Then for $z \in \Omega+1$ and $\tau \in S_{b}$ we have

$$
-\tau(-z)^{b}=-|\tau||z|^{b} \exp (i(b \arg (-z)+\arg \tau)) .
$$

Thus

$$
\left|\exp \left(-\tau(-z)^{b}\right)\right|=\exp \left(-|\tau||z|^{b} \cos (b \arg (-z)+\arg \tau)\right),
$$

which by

and

$$
\limsup _{|z| \rightarrow \infty, z \in \Omega+1} b \arg (-z)+\arg \tau=\frac{\pi(1+b)}{4}
$$

can be estimated by

$$
\liminf _{|z| \rightarrow \infty, z \in \Omega+1} b \arg (-z)+\arg \tau=-\frac{\pi(1+b)}{4}
$$

$$
\text { const } \cdot \exp \left(-|\tau||z|^{b} \cos (\pi(3+b) / 8)\right) \text {. }
$$

So the integrand in (17) can be estimated by

$$
C^{\prime} \exp \left(-|\tau||\lambda|^{b} \cos (\pi(3+b) / 8)\right)(1+|\lambda|)^{k}
$$

which proves the existence of the integral. Thus $C_{b, \tau} \in L(E)$. Clearly $C_{b, \tau}$ commutes with $A$, and we have

$$
A C_{b, \tau}=\frac{1}{2 \pi i} \int_{\Gamma} e^{-\tau(-\lambda)^{b}} A R(\lambda, A) d \lambda=\frac{1}{2 \pi i} \int_{\Gamma} e^{-\tau(-\lambda)^{b}} \lambda R(\lambda, A) d \lambda
$$

by the resolvent equation and by the equality

$$
\frac{1}{2 \pi i} \int_{\Gamma} e^{-\tau(-\lambda)^{b}} d \lambda=0
$$

which we get by Cauchy's theorem and a standard estimate. 
Letting $m \in \mathbb{N}$ we get from (19) by induction

$$
A^{m} C_{b, \tau}=\frac{1}{2 \pi i} \int_{\Gamma} e^{-\tau(-\lambda)^{b}} \lambda^{m} R(\lambda, A) d \lambda,
$$

and thus $A^{m} C_{b, \tau} \in L(E)$ by the estimation (18) of the integrand, so $\operatorname{Im} C_{b, \tau} \subset$ $D\left(A^{m}\right)$. Since $m \in \mathbb{N}$ was arbitrary, this implies $\operatorname{Im} C_{b, \tau} \subset D_{\infty}(A)$.

Now we have to show the injectiveness of the operators $C_{b, \tau}$ which follows from Lemma 3 in [5] or can be shown by using Theorem II-3 and Lemma II-1 from [3]. For the convenience of the reader we will sketch the proof.

Lemma 5.4. For all $\tau \in S_{b}$ the operator $C_{b, \tau}$ is injective.

Proof. In a first step we show

$$
\lim _{\tau \rightarrow 0, \tau \in S_{b}} C_{b, \tau} x=x
$$

for all $x \in D\left(A^{k+2}\right)$ (see [5], Lemma 1, and [3], Lemma II-1). So let $x \in D\left(A^{k+2}\right)$ and $y:=A^{(k+2)} x$. Then

$$
C_{b, \tau} x=\frac{1}{2 \pi i} \int_{\Gamma} \frac{\exp \left(-\tau(-\lambda)^{b}\right)}{\lambda^{k+2}} R(\lambda, A) y d \lambda
$$

for all $\tau \in S_{b}$. Thus by the dominated convergence theorem

$$
\lim _{\tau \rightarrow 0, \tau \in S_{b}} C_{b, \tau} x=\frac{1}{2 \pi i} \int_{\Gamma} \frac{R(\lambda, A) y}{\lambda^{k+2}} d \lambda .
$$

We deform the curve of integration $\Gamma$ to $\partial K(0, \delta)$, where we choose $\delta$ such that $K(0, \delta) \subset \Lambda$. Since by Cauchy's formula

$$
\frac{1}{2 \pi i} \int_{\Gamma} \frac{R(\lambda, A) y}{\lambda^{k+2}} d \lambda=\frac{1}{2 \pi i} \int_{\partial K(0, \delta)} \frac{R(\lambda, A) y}{\lambda^{k+2}} d \lambda=A^{-(k+2)} y=x,
$$

the first step is finished.

In a second step we show that $\left(C_{b, \tau}\right)_{\tau \in S_{b}}$ is a holomorphic semigroup (see [5], Lemma 2, and [3], Theorem II-3). Since the integrand in (17) is holomorphic in $\tau \in S_{b}$ and vanishes with all derivatives sufficiently rapidly in $\infty$ on $\Gamma$, we can interchange differentiation and integration and the mapping $S_{b} \rightarrow L(E), \tau \mapsto C_{b, \tau}$ is holomorphic. Let now $\sigma, \tau \in S_{b}$ and $\Gamma^{\prime}:=\Gamma+1 / 2$. In (17) for $C_{b, \sigma}$ we can deform the curve of integration to $\Gamma^{\prime}$. Now by the resolvent equation

$$
\begin{aligned}
C_{b, \sigma} C_{b, \tau}= & \frac{1}{(2 \pi i)^{2}} \int_{\Gamma^{\prime}} \int_{\Gamma} \exp \left(-\sigma(-\lambda)^{b}\right) \exp \left(-\tau(-\mu)^{b}\right) R(\lambda, A) R(\mu, A) d \mu d \lambda \\
= & \frac{1}{(2 \pi i)^{2}} \int_{\Gamma^{\prime}} \exp \left(-\sigma(-\lambda)^{b}\right) R(\lambda, A)\left(\int_{\Gamma} \frac{\exp \left(-\tau(-\mu)^{b}\right)}{\mu-\lambda} d \mu\right) d \lambda \\
& -\frac{1}{(2 \pi i)^{2}} \int_{\Gamma} \exp \left(-\tau(-\mu)^{b}\right) R(\mu, A)\left(\int_{\Gamma^{\prime}} \frac{\exp \left(-\sigma(-\lambda)^{b}\right)}{\mu-\lambda} d \lambda\right) d \mu
\end{aligned}
$$

The first term in brackets vanishes since the integrand is holomorphic on the left of $\Gamma$. The second term in brackets equals $-\exp \left(-\sigma(-\mu)^{b}\right)$ by Cauchy's formula. Thus

and the second step is finished.

$$
C_{b, \sigma} C_{b, \tau}=C_{b, \sigma+\tau}
$$

For the third step let $\tau \in S_{b}$ and $y \in \operatorname{Kern} C_{b, \tau}$. We will show $y=0$. Let $x:=A^{-(k+2)} y$. Then $x \in \operatorname{Kern} C_{b, \tau}$ since $C_{b, \tau}$ commutes with $A^{-1}$. By the second 
step the holomorphic mapping $S_{b} \rightarrow E, \sigma \mapsto C_{b, \sigma} x$ vanishes on $\tau+S_{b}$, so it vanishes everywhere. But then by the first step $x=0$, thus $y=0$, and $C_{b, \tau}$ is injective.

We are now ready to prove

Theorem 5.5. Let $b \in] 0,1\left[\right.$ and $\tau \in S_{b}$. Then $A$ generates a $C_{b, \tau}$-regularized semigroup.

Proof. By Lemmas 5.3 and 5.4 the operator $C_{b, \tau}$ is bounded, injective, commutes with $A$ and satisfies $\operatorname{Im} C_{b, \tau} \subset D_{\infty}(A)$. Since $A$ generates a DSG by Corollary 4.8 (14), the Cauchy problem

$$
u^{\prime}(t)=A u(t), \quad t \geq 0, \quad u(0)=x
$$

has a unique mild solution for all $x \in D_{\infty}(A)$. Thus $A$ generates a $C_{b, \tau}$-regularized semigroup by Proposition 5.2, since $A$ has non-empty resolvent set.

Remark 5.6. We remark that for densely defined $A$ Theorem 5.5 is a consequence of Corollary 3.12 and [11], Theorem 5.2, by letting $h(z):=(r-z)^{n+2} \exp \left(-\tau(-z)^{b}\right)$ for a suitable $r \in \rho(A)$ and $V:=\mathbb{C} \backslash \Lambda$, and follows also by the proof of [4], Theorem 1 '.

The converse of Theorem 5.5 is not true as can be seen from the work of Beals ([5]), and it seems to be an open problem to give a characterization of the generators of distribution semigroups in terms of regularized semigroups.

After this paper had been accepted the author learned that the definition of a DSG, i.e. (3) and (4), as well as the assertions of Corollary 4.8 and Theorem 4.11 also appear in [25].

\section{REFERENCES}

1. W. Arendt, Vector-valued Laplace transforms and Cauchy problems, Israel J. Math. 59 (1987), 327-352. MR 89a:47064

2. W. Arendt, O. El-Mennaoui, V. Keyantuo, Local Integrated Semigroups: Evolution with Jumps of Regularity, J. Math. Analysis Appl. 186 (1994), 572-595. MR 95f:47065

3. M. Balabane, Puissances fractionnaires d'un opérateur générateur d'un semi-groupe distribution régulier, Ann. Inst. Fourier, Grenoble, 26 (1976), 157-203. MR 53:6353

4. R. Beals, On the abstract Cauchy problem, J. Funct. Analysis 10 (1972), 281-299. MR 51:8859

5. R. Beals, Semigroups and abstract Gevrey spaces, J. Funct. Analysis 10 (1972), 300-308. MR 50:14355

6. J. Chazarain, Problèmes de Cauchy abstraits et applications à quelques problèmes mixtes, $J$. Funct. Analysis 7 (1971), 386-446. MR 43:2570

7. G. Da Prato, E. Sinestrari, Differential Operators with Non-dense Domain, Ann. Scuola Normale Pisa 14 (1987), 285-344. MR 89f:47062

8. E. B. Davies, M. M. H. Pang, The Cauchy Problem and a Generalization of the Hille-Yosida Theorem, Proc. London Math. Soc. 55 (1987), 181-208. MR 88e:34100

9. R. deLaubenfels, $C$-existence families and improperly posed problems, Semesterbericht Funktionalanalysis, Tübingen WS 1989/90, 155-171.

10. R. deLaubenfels, "Existence Families, Functional Calculi and Evolution Equations," Lect. Notes in Math. 1570, Springer, 1994. MR 96b:47047

11. R. deLaubenfels, Automatic extension of functional calculi, Stud. Math. 114 (1995), 237-259. MR 96f: 47029

12. H. O. Fattorini, Some remarks on convolution equations for vector valued distributions, Pacific J. Math. 66 (1976), 347-371. MR 57:13471

13. H. O. Fattorini, Vector valued distributions having a smooth convolution inverse, Pacific J. Math. 90 (1980), 347-372. MR 82f:46046

14. H. O. Fattorini, The Cauchy problem, Addison-Wesley, 1983. MR 84g:34003 
15. V. Keyantuo, Semi-groupes distributions, semi-groupes intrégrés et problèmes d'évolution, Thèse, Université de Franche-Comté, Besançon 1992.

16. J. L. Lions, Les semi-groupes distributions, Portugal. Math. 19 (1960), 141-164. MR 26:611

17. Yu. I. Lyubich, Investigation of the deficiency of the abstract Cauchy problem, Soviet Math. Dokl. 7 (1960), 166-169.

18. I. Miyadera, M. Okubo, N. Tanaka, On Integrated Semigroups which are not Exponentially Bounded, Proc. Japan Acad., Ser. A 69 (1993), 199-204. MR 95a:47037

19. L. Schwartz, Théorie des distributions, Hermann, Paris, 1966. MR 35:730

20. L. Schwartz, Théorie des distributions à valeurs vectorielles, I: Ann. Inst. Fourier 7 (1957), 1-141; II: Ann. Inst. Fourier 8 (1958), 1-209. MR 21:6534; MR 22:8322

21. R. Shiraishi, Y. Hirata, Convolution Maps and Semi-group Distributions, J. Sci. Hiroshima Univ. Ser. A-I 28 (1964), 71-88. MR 30:1396

22. N. Tanaka, I. Miyadera, C-Semigroups and the abstract Cauchy problem, J. Math. Analysis Appl. 170 (1992), 196-206. MR 93j:47061

23. N. Tanaka, N. Okazawa, Local $C$-semigroups and local integrated semigroups, Proc. London Math. Soc. 61 (1990), 63-90. MR 91b:47093

24. H. R. Thieme, Integrated semigroups and integrated solutions to abstract Cauchy problems, J. Math. Analysis Appl. 152 (1990), 416-447. MR 91k:47093

25. S. Wang, A kind of smooth distribution semigroups and integrated semigroups, J. Funct. Analysis, to appear.

Mathematisches Seminar der Christian-Albrechts-Universität Zu Kiel, LudewigMeyn-Strasse 4, D-24098 Kiel, Germany

Current address: Mathematisches Institut I der Universität Karlsruhe, Englerstraße 2, D-76128 Karlsruhe, Germany

E-mail address: peer.kunstmann@math.uni-karlsruhe.de 